\title{
Cogitare
Enfermagem
}

\section{INCIDÊNCIA DE VOLUME DE LÍQUIDOS EXCESSIVO EM PACIENTES ADULTOS SOB CUIDADOS INTENSIVOS}

\author{
Caroline Monteiro Bittencourt ${ }^{1}$ (i) \\ Josefine Busanello ${ }^{1}$ (i) \\ Jenifer Harter ${ }^{1}$ (1) \\ Raquel Potter Garcia' ${ }^{1}$
}

\begin{abstract}
RESUMO
Objetivo: identificar a incidência do diagnóstico de enfermagem Volume de Líquidos Excessivo em pacientes internados em Unidade de Terapia Intensiva, no período de três meses.

Método: estudo transversal, descritivo e exploratório, em Unidade de Terapia Intensiva da região sul do Brasil. Foram analisadas as características clínicas obtidas no prontuário e exame físico de 31 pacientes, admitidos entre março e maio de 2018, por distribuição de frequência, e análise bivariada com teste Qui-quadrado.

Resultados: $54,8 \%$ dos pacientes desenvolveram Volume de Líquidos Excessivo, prevalecendo as características definidoras: edema, hemoglobina e hematócrito diminuídos, anasarca e desequilíbrio eletrolítico. Comprometimento neurológico, ventilação mecânica, sedação e mais de quatro dias de internação foram evidenciados como principais fatores relacionados.

Considerações finais: este estudo contribui para a acurácia diagnóstica do Volume de Líquidos Excessivo dos pacientes internados em terapia intensiva e amplia a possibilidade de desenvolvimento deste diagnóstico de enfermagem.
\end{abstract}

DESCRITORES: Diagnóstico de Enfermagem; Incidência; Líquidos Corporais; Deslocamentos de Líquidos Corporais; Unidades de Terapia Intensiva.

\section{INCIDENCIA DE VOLUMEN LÍQUIDOS EXCESIVO EN PACIENTES ADULTOS BAJO CUIDADOS INTENSIVOS}

\section{RESUMEN:}

Objetivo: identificar la incidencia del diagnóstico de enfermería Volumen Líquido Excesivo em pacientes ingresados en una Unidad de Cuidados Intensivos durante un periodo de tres meses. Método: estudio transversal, descriptivo y exploratorio, en una Unidad de Cuidados Intensivos del sur de Brasil. Las características clínicas obtenidas en registros médicos y exámenes físicos de 31 pacientes, ingresados entre marzo y mayo de 2018 se analizaron mediante la distribución de frecuencias y el análisis bivariado con el test de Chi-cuadrado. Resultados: El 54,8\% de los pacientes desarrollaron un Volumen Líquido Excesivo, predominando las características definitorias: edema, hemoglobina y hematocrito disminuidos, anasarca y desequilibrio electrolítico. El deterioro neurológico, la ventilación mecánica, la sedación y más de cuatro días de hospitalización se evidenciaron como los principales factores relacionados. Consideraciones finales: este estudio contribuye a la precisión diagnóstica del Volumen Líquido Excesivo en pacientes hospitalizados en cuidados intensivos y amplía la posibilidad de desarrollar este diagnóstico de enfermería.

DESCRIPTORES: Diagnóstico de Enfermería; Incidencia; Líquidos Corporales; Transferencias de Fluidos Corporales; Unidades de Cuidados Intensivos. 
No contexto complexo de uma Unidade de Terapia Intensiva (UTI), o enfermeiro é fundamental dentro da equipe multiprofissional, uma vez que está comprometido com a assistência direta e ininterrupta a pacientes em situações críticas de saúde ${ }^{(1)}$. A fim de sistematizar essa assistência, o enfermeiro pode e deve utilizar o Processo de Enfermagem, que configura uma ferramenta qualificadora do cuidado ao reduzir erros e altear a segurança do paciente ${ }^{(2)}$. Entre as taxonomias que padronizam a linguagem do Processo de Enfermagem, está a NANDA-I, que traz os Diagnósticos de Enfermagem (DE).

Um dos DE prevalentes em UTls é o Volume de Líquidos Excessivo (VLE)(3), que foi aprovado em 1982 e revisado em 1996, 2013 e 2017. Apresenta nível de evidência 2.1, classificação necessária para aceite de inclusão na taxonomia NANDA-I, a partir da avaliação de todos os componentes (título, definição, características definidoras, fatores relacionados ou de risco) e também da literatura. Ele faz parte do domínio 2 - Nutrição, em sua classe 5 - Hidratação, tendo como definição "entrada excessiva e/ou retenção de líquidos"(4).

Uma vez que o DE é a base para determinar o plano de cuidados, deve-se priorizar aqueles que alcancem os resultados esperados acerca da condição clínica do paciente. Também é pertinente considerar DE similares ou cujos sinais e sintomas são muito semelhantes para que não haja uma baixa acurácia diagnóstica(4). Especificamente, em relação ao VLE, indicativos clínicos de alteração da pressão arterial e do padrão respiratório, e inquietação, podem caracterizar outros $\mathrm{DE}$, tais como Padrão Respiratório Ineficaz e Débito cardíaco diminuído, e prejudicar a acurácia diagnóstica.

Nesta perspectiva, tencionou-se estudar o DE VLE em pacientes internados em UTI, em virtude de sua frequência nesse contexto, verificada a partir da vivência clínica e pela incipiência de pesquisas que abordem essa temática e que subsidiem o Enfermeiro para a acurácia diagnóstica. Sobretudo, em razão de a sobrecarga hídrica ter efeitos deletérios à saúde, pois aumenta a instabilidade hemodinâmica do paciente, pode causar congestão de órgãos e, inclusive, evoluir para óbitoo(5-6).

Destarte, o objetivo deste estudo foi identificar a incidência do diagnóstico de enfermagem VLE em pacientes internados em Unidade de Terapia Intensiva, no período de três meses, e também identificar as características definidoras e os fatores relacionados prevalentes nos pacientes com VLE.

\section{MÉTODO}

Estudo transversal, descritivo e exploratório realizado em uma UTI Adulta da região sul do Brasil, entre os meses de março a maio de 2018. Nessa unidade, a média mensal é de 11 internações. Para o cálculo amostral, considerou-se a população de 33 pacientes tendo em vista o período de três meses do estudo, com nível de confiança de $95 \%$ e margem de erro de $5 \%$, obtendo uma amostra de 31 pacientes. O critério para composição da amostra foi pacientes maiores de 18 anos em situação crítica de vida. Foram excluídos da amostra pacientes que não completaram 24 horas de internação na UTI, devido a óbito ou transferência para outra unidade.

A coleta de dados sucedeu-se por duas etapas guiadas por um instrumento semiestruturado. Os pacientes foram avaliados e acompanhados por no máximo 10 dias. O tempo máximo de acompanhamento foi estabelecido com a realização de um estudo 
piloto, que demonstrou que o DE em estudo foi manifestado antes de 10 dias de internação. O estudo piloto também permitiu a averiguação das variáveis do instrumento de coleta de dados e do protocolo pré-estabelecido para o alcance do objetivo.

A primeira etapa foi a análise dos prontuários dos pacientes, com ênfase para variáveis demográficas (sexo, idade, estado civil, profissão/ocupação, naturalidade, escolaridade), caracterização clínica (antecedentes/comorbidades, alergias, diagnóstico médico, diagnósticos de enfermagem, fatores relacionados ao DE VLE, características definidoras do DE VLE, tempo de internação, desfecho), exames complementares, terapia medicamentosa, dispositivos invasivos utilizados, intervenções de enfermagem. O exame físico diário do paciente constituiu a segunda etapa e estava voltado para avaliar o estado geral de saúde do paciente, verificar a presença de sinais e sintomas do diagnóstico de enfermagem VLE e a presença de dispositivos invasivos.

Os dados obtidos foram organizados em planilha eletrônica no programa Excel ( 2010 e, posteriormente, analisados com o software Statistical Package for Social Sciences $₫$ (SPSS) versão 20.0. Empregou-se a estatística descritiva com frequências absolutas e relativas. Realizou-se análise multivariável a fim de identificar fatores risco para VLE. Variáveis que foram consideradas associadas a VLE (edema, hemoglobina e hematócrito diminuídas, distúrbio eletrolítico, anasarca, sedação, ventilação mecầnica, uso de diurético, reposição de eletrólitos, balanço hídrico acumulado e desfecho) foram incluídas na análise multivariável, realizando-se, desta forma, o ajuste dos referidos fatores para a identificação de fatores preditores de VLE, minimizando-se assim o viés de confundimento no estudo. Utilizou-se o teste Qui-quadrado para averiguar a associação das variáveis categóricas. Assumiu-se um nível de significância bilateral do valor $p<0,05$.

Para elencar o DE, adotou-se a orientação da NANDA-I de manifestação mínima de quatro características definidoras para acurar a escolha e diferenciar diagnósticos semelhantes, evitando as mais comuns que caracterizam vários $\mathrm{DE}$, como alteração da pressão arterial, alteração do padrão respiratório e inquietação.

Os pacientes e/ou familiares foram convidados a participar, clarificados sobre 0 estudo e inclusos mediante assinatura do Termo de Consentimento Livre e Esclarecido. Foram respeitados os preceitos éticos da Resolução 466/2012 do Conselho Nacional de Saúde, com a anuência do Comitê de Ética em Pesquisa da instituição, sob o parecer $n$. 2.424.231.

\section{RESULTADOS}

Foram avaliados 31 pacientes. Considerando as características demográficas, a maioria era do sexo masculino $(58,1 \% ; n=17)$, os casados somavam $48,4 \%$ ( $n=15)$ e $32,3 \%$ $(n=10)$ eram aposentados. A média de idade foi de $59,5 \pm 16,75$ anos, com variabilidade de 22 anos a 84 anos.

Quanto à procedência dos pacientes, a maioria $(54,8 \% ; n=17)$ era da Unidade de Emergência, seguidos daqueles provenientes do Bloco Cirúrgico cinco $(16,1 \%)$ e transferidos de outro hospital cinco (16,1\%). Minoria proveniente da Unidade de Internação Clínica ou Cirúrgica quatro (13\%). Quanto ao prognóstico, $58,1 \%(n=18)$ deram alta da UTI para outra unidade de internação, sete $(22,6 \%)$ seguiram internados por mais de 10 dias na UTI, quatro $(12,9 \%)$ foram a óbito e dois $(6,5 \%)$ foram transferidos para outra instituição.

Os diagnósticos médicos dos pacientes avaliados resultaram de afecções que comprometem, principalmente, os sistemas orgânicos: neurológico 41,9\% $(n=13)$, respiratório cinco $(16,1 \%)$ e cardiológico quatro (12,9\%). Os DE prioritários que prevaleceram na admissão na UTI foram: Risco de perfusão tissular cerebral ineficaz $16(51,6 \%)$, Padrão 
respiratório ineficaz cinco (16,1\%), Intolerância à atividade cinco (16,1\%), Troca de gases prejudicada três $(9,7 \%)$, e Dor aguda dois $(6,5 \%)$.

Além das afecções responsáveis pela admissão na UTI, alguns pacientes apresentaram condições associadas, ou seja, comorbidades, que podem colaborar para o desenvolvimento do DE VLE. Essas comorbidades envolvem mecanismos de regulação comprometidos e foram subdivididas para melhor apresentação. O somatório apresentado ultrapassa os $100 \%$ pelo fato de alguns pacientes apresentarem mais de um mecanismo de regulação comprometido. Ainda, considerando o momento da admissão, identificou-se que apenas um paciente apresentou o fator relacionado excesso de líquidos e dois apresentaram fator relacionado excesso de sódio. Esses dados constam na Tabela 1.

Tabela 1 - Componentes do Diagnóstico de Enfermagem Volume de Líquidos Excessivo manifestados pelos pacientes. Uruguaiana, RS, Brasil, 2018

\begin{tabular}{|c|c|c|c|}
\hline \multirow{3}{*}{$\begin{array}{l}\text { Fator } \\
\text { relacionado }\end{array}$} & & \multirow{2}{*}{$\frac{\mathbf{n}}{1}$} & \multirow{2}{*}{$\begin{array}{c}\% \\
3,2 \\
\end{array}$} \\
\hline & Excesso de líquidos & & \\
\hline & Excesso de sódio & 2 & 6,4 \\
\hline \multirow{3}{*}{$\begin{array}{l}\text { Condição } \\
\text { associada }\end{array}$} & Mecanismo regulador comprometido: função cardiovascular & 18 & 58 \\
\hline & Mecanismo regulador comprometido: função metabólica & 9 & 29 \\
\hline & Mecanismo regulador comprometido: função renal & 2 & 6,4 \\
\hline \multicolumn{2}{|c|}{ Nenhum fator relacionado ou condição associada } & 8 & 25,8 \\
\hline \multirow{20}{*}{$\begin{array}{l}\text { Características } \\
\text { definidoras }\end{array}$} & Alteração do estado mental & 22 & 71 \\
\hline & Ruídos adventícios & 20 & 64,5 \\
\hline & Diminuição da hemoglobina & 19 & 61,3 \\
\hline & Diminuição do hematócrito & 19 & 61,3 \\
\hline & Alteração no padrão respiratório & 19 & 61,3 \\
\hline & Dispneia & 18 & 58,1 \\
\hline & Alteração da pressão arterial & 18 & 58,1 \\
\hline & Edema & 18 & 58,1 \\
\hline & Ingestão maior que eliminação & 16 & 51,6 \\
\hline & Azotemia & 14 & 45,2 \\
\hline & Ansiedade & 13 & 41,9 \\
\hline & Desequilíbrio eletrolítico & 10 & 32,2 \\
\hline & Inquietação & 8 & 25,8 \\
\hline & Ortopneia & 7 & 22,6 \\
\hline & Congestão pulmonar & 6 & 19,4 \\
\hline & Presença de B3 & 6 & 19,4 \\
\hline & Anasarca & 5 & 16,1 \\
\hline & Distensão da veia jugular & 4 & 12,9 \\
\hline & Hepatomegalia & 4 & 12,9 \\
\hline & Oligúria & 3 & 9,7 \\
\hline
\end{tabular}


Dentre as 27 características definidoras do VLE, houve a impossibilidade de mensuração de quatro delas: alteração na gravidade específica da urina, alteração da pressão arterial pulmonar, aumento da pressão venosa central e ganho de peso em curto período. Contudo, tal fragilidade não impactou na definição do DE VLE. Três características definidoras não foram manifestadas: derrame pleural, dispneia paroxística noturna e reflexo hepatojugular positivo. Outros sinais e sintomas foram evidenciados na amostra, conforme a Tabela 1.

Na análise entre o tempo de internação na UTI e outras variáveis, evidenciou-se que quanto maior o tempo de internação na UTI, maior a probabilidade de desenvolver VLE e maior a necessidade de cuidados intensivos. Estes dados são apresentados na Tabela 2. Destaca-se que a existência de uma associação temporal que reforça a evidência para a exposição de tempo, acima de quatro dias de internação na UTI e maior prevalência acima de oito dias, ser uma causa possível do desfecho estudado, o DE VLE.

Tabela 2 - Associação de variáveis independentes com o tempo de internação na UTI. Uruguaiana, RS, Brasil, 2018

Variáveis

Tempo de internação na UTI

\begin{tabular}{|c|c|c|c|c|c|c|c|c|c|}
\hline & \multicolumn{2}{|c|}{ Até 3 dias } & \multicolumn{2}{|c|}{4 a 7 dias } & \multicolumn{2}{|c|}{8 a 10 dias } & \multicolumn{2}{|c|}{ Total } & \multirow[t]{2}{*}{ Valor de $p$} \\
\hline & $\mathbf{n}$ & $\%$ & $\mathbf{N}$ & $\%$ & $\mathbf{n}$ & $\%$ & $\mathbf{n}$ & $\%$ & \\
\hline $\begin{array}{l}\text { Pacientes com } \\
\text { comprometimento do } \\
\text { sistema neurológico no } \\
\text { momento da internação }\end{array}$ & 2 & 6,5 & 4 & 13 & 12 & 38,7 & 18 & 58 & 0,203 \\
\hline $\begin{array}{l}\text { Pacientes com balanço } \\
\text { hídrico acumulado } \\
\text { positivo }\end{array}$ & 5 & 16 & 6 & 19,4 & 7 & 22,6 & 18 & 58 & 0,367 \\
\hline Pacientes com alta da UTI & 5 & 16 & 9 & 29 & 4 & 12,9 & 18 & 58 & 0,017 \\
\hline $\begin{array}{l}\text { Pacientes que foram a } \\
\text { óbito }\end{array}$ & 1 & 3,2 & 2 & 6,5 & 1 & 3,2 & 4 & 12,9 & 0,017 \\
\hline $\begin{array}{l}\text { Pacientes que } \\
\text { desenvolveram VLE }\end{array}$ & 1 & 3,2 & 4 & 13 & 12 & 38,7 & 17 & 54,8 & 0,005 \\
\hline $\begin{array}{l}\text { Pacientes em ventilação } \\
\text { mecânica }\end{array}$ & 2 & 6,5 & 4 & 12,9 & 12 & 38,7 & 18 & 58 & 0,018 \\
\hline Pacientes com sedação & 2 & 6,5 & 3 & 9,7 & 13 & 42 & 18 & 58 & 0,002 \\
\hline
\end{tabular}

Fonte: Autores (2018).

A média de acompanhamento dos pacientes para coleta de dados foi de 6,1 dias, com mediana de sete dias, e com variabilidade mínima de dois dias e máxima de 10 dias. Durante esse período, 17 pacientes desenvolveram VLE. Portanto, a incidência global foi de $54,8 \%$. No que concerne o dia de desenvolvimento do VLE, nenhum paciente manifestou quatro características definidoras no primeiro dia de internação, três manifestaram no segundo dia, três no terceiro dia, sete no quarto dia, dois no quinto dia, um no sétimo dia e um no oitavo dia.

Ainda, em relação aos resultados apresentados na Tabela 2, os pacientes que 
sobreviveram na UTI tiveram alta, predominantemente, entre o quarto e sétimo dia de internação (29\%; $n=9)$. O desfecho óbito acometeu $12,9 \%(n=4)$ pacientes avaliados no estudo. Outros achados, mesmo sem significância estatística, frente à associação de variáveis independentes com o tempo de internação na UTI, apresentam relevância. Destaca-se que $58 \%(n=18)$ dos pacientes avaliados apresentaram comprometimento do sistema neurológico no momento da internação, e destes $38,7 \%(n=12)$ com tempo de internação superior a oito dias. Considerando o balanço hídrico acumulado positivo, $16 \%$ dos pacientes apresentaram esta condição volêmica já nos primeiros três dias de internação.

Constatou-se associação entre o desenvolvimento de VLE e as características definidoras: edema, hemoglobina e hematócrito diminuídos, anasarca e desequilíbrio eletrolítico; demonstrando que estes sinais são indicativos do diagnóstico em pacientes graves. A ventilação mecânica e a sedação foram evidenciadas como fatores de risco para o desenvolvimento do diagnóstico. $O$ comprometimento neurológico no momento da internação, pacientes com alta da UTI, pacientes que foram a óbito, pacientes que permaneceram internados na UTI, pacientes transferidos para outras instituiçõos também tiveram associação com o desenvolvimento de VLE, conforme demonstra a Tabela 3.

Tabela 3 - Análise multivariada para identificação das características definidoras e fatores relacionados a Volume de Líquidos Excessivo em paciente internado em UTI. Uruguaiana, RS, Brasil, 2018

\begin{tabular}{|c|c|c|c|c|c|c|c|c|}
\hline \multirow[t]{2}{*}{ Variáveis } & \multicolumn{2}{|c|}{$\begin{array}{c}\text { Pacientes com } \\
\text { VLE }\end{array}$} & \multicolumn{2}{|c|}{ Total } & \multirow[t]{2}{*}{$\begin{array}{l}\text { Valor } \\
\text { de } p\end{array}$} & \multirow[t]{2}{*}{ OR } & \multicolumn{2}{|c|}{ IC: $95 \%$} \\
\hline & $\mathbf{n}$ & $\%$ & $\mathbf{n}$ & $\%$ & & & Min & $\operatorname{Max}$ \\
\hline Edema & 15 & 83,3 & 18 & 58,1 & 0 & 27,5 & 3,908 & 193,489 \\
\hline $\begin{array}{l}\text { Hemoglobina e hematócrito } \\
\text { diminuída }\end{array}$ & 16 & 84,2 & 19 & 61,3 & 0 & 58,667 & 5,377 & 640,146 \\
\hline Desequilíbrio eletrolítico & 9 & 90 & 10 & 32,3 & 0,007 & 14,625 & 1,548 & 138,187 \\
\hline Anasarca & 5 & 100 & 5 & 16,1 & 0,027 & 2,167 & 1,43 & 3,282 \\
\hline Ventilação mecânica & 13 & 72,2 & 18 & 58,1 & 0,022 & 5,85 & 1,222 & 27,994 \\
\hline Sedação & 14 & 77,8 & 18 & 58,1 & 0,003 & 11,667 & 2,125 & 64,039 \\
\hline Soroterapia contínua & 16 & 59,3 & 27 & 87,1 & 0,199 & 4,364 & 0,4 & 0,199 \\
\hline Reposição de eletrólitos & 3 & 75,5 & 4 & 12,9 & 0,385 & 2,786 & 0,256 & 30,273 \\
\hline Uso de diurético & 8 & 66,7 & 12 & 38,7 & 0,293 & 2,222 & 0,496 & 9,964 \\
\hline $\begin{array}{l}\text { Balanço hídrico acumulado } \\
\text { positivo }\end{array}$ & 9 & 50 & 18 & 58,1 & 0,524 & 0,625 & 0,147 & 2,664 \\
\hline Pacientes com alta da UTI & 5 & 27,8 & 18 & 58,1 & 0,004 & - & - & - \\
\hline Óbito & 3 & 75 & 4 & 13 & 0,004 & & & \\
\hline Permaneceu internado na UTI & 7 & 100 & 7 & 22,6 & 0,004 & - & - & - \\
\hline $\begin{array}{l}\text { Transferência para outra } \\
\text { instituição }\end{array}$ & 2 & 100 & 2 & 6,5 & 0,004 & - & - & - \\
\hline
\end{tabular}

$\begin{aligned} & \text { Comprometimento do } \\ & \text { sistema neurológico no } \\ & \text { momento da internação }\end{aligned}$
Legenda: VLE: volume de líquidos excessivo; OR: Odd Ration; IC: intervalo de confiança; Min: valor mínimo; Max: valor máximo.
Fonte: Autores (2018).


Observando a Tabela 2 e a Tabela 3, percebe-se que a soroterapia contínua, a reposição de eletrólitos, o uso de diuréticos e o balanço hídrico acumulado positivo não tiveram associação direta com o tempo de internação na UTI ou com o desenvolvimento de VLE, também não sendo evidenciado este como um fator de risco para o diagnóstico. Já a sedação e a ventilação mecânica tiveram associação significativa com tempo de internação na UTI e, também, com o desenvolvimento de VLE. Ademais, o comprometimento neurológico na admissão na UTI não teve associação com o tempo de internação na UTI, mas teve com o desenvolvimento de VLE.

\section{DISCUSSÃO}

Similarmente ao apresentado na literatura, o perfil demográfico e clínico dos pacientes demonstrou que a maioria é do sexo masculino e com a média de idade ultrapassando os 55 anos. O principal diagnóstico médico identificado entre os pacientes está relacionado a afecções neurológicas, a maioria é proveniente da unidade de emergência e predominantemente, os pacientes deram alta da UTI para outra unidade de internação(7).

Pacientes neurológicos podem apresentar distúrbios hidroeletrolíticos por diversos motivos, como administração de diuréticos osmóticos, infusão de fluidos, disfunção da hipófise e aumento da perda hídrica insensível. Em vista disso, deve-se considerar todas as variáveis durante a avaliação clínica, monitorar os níveis séricos de eletrólitos e registrar rigorosamente a ingestão e a eliminação hídrica, com o intuito de manter os níveis de estabilidade ${ }^{(8)}$.

Vale ressaltar que a instituição onde foi realizada a pesquisa é referência regional em neurologia, o que justifica uma admissão maior de pacientes neurológicos na UTI em questão. Em consequência, o DE mais elencado na admissão foi Risco de perfusão tissular cerebral ineficaz. Salienta-se, ainda, que não foram elencados diagnósticos considerados inerentes a pacientes críticos, como Risco de infecção, que é condicionado pela exposição a procedimentos invasivos ${ }^{(9)}$.

O fator comprometimento de mecanismos reguladores ocorre quando há falhas no $\mathrm{pH}$ dos fluidos corpóreos, na pressão osmótica e na equidade entre cátions e ânions, provocando desequilíbrio entre ingestão e eliminação de água e eletrólitos ${ }^{(10)}$. Nessas circunstâncias, enquadram-se pacientes com insuficiência renal, problemas cardiovasculares e com insuficiência cardíaca, que desenvolvem VLE e necessitam de cuidados para evitar e controlar tal problema(11).

O fator ingestão ou administração excessiva de líquidos indica que o paciente está recebendo uma quantidade de líquidos maior que a metabolizada. O fator ingestão ou administração excessiva de sódio pode ser resultado de uma dieta inadequada ou reposição volểmica com soro fisiológico 0,9\%, desencadeando a retenção de líquidos, sobrecarregando o sistema renal, e dificultando a filtração e a eliminação de líquidos ${ }^{(11)}$. Como apenas um paciente foi identificado com excesso de líquidos e dois com excesso de sódio ao serem admitidos na UTI, infere-se que esses fatores não são a causa para o desenvolvimento do VLE neste estudo. Quanto às características definidoras para elencar - VLE, esta pesquisa coincide parcialmente com outro importante estudo que aduz que as mais utilizadas são: oligúria, ingesta maior que a eliminação, mudança no padrão respiratório, dispneia, ortopneia, ruídos respiratórios adventícios, congestão pulmonar, ganho de peso em um curto período, edema e anasarca ${ }^{(11)}$.

A elevada alteração no estado mental pode ser um dado tendencioso, tendo em vista que a maioria dos pacientes apresentou comprometimento neurológico já na admissão na UTI. A alteração no padrão respiratório, a presença de ruídos adventícios e dispneia nem sempre estão associadas a doenças de base, podendo ser consequência da congestão 
pulmonar causada pelo excesso de líquidos ${ }^{(6)}$.

O paciente em situação crítica de vida é submetido a terapias intravenosas que, se não forem devidamente controladas, podem conduzir a hipervolemia, devido à entrada de fluidos ser maior que a sua eliminação. A alteração da volemia pode desencadear desequilíbrio eletrolítico que compromete o desempenho cardíaco, posto que este depende de eletrólitos para seu funcionamento ${ }^{(12)}$.

Além do desequilíbrio eletrolítico, a diminuição de hematócrito e hemoglobina é um sinal corrente da hipervolemia(13-14), causado pela hemodiluição, ou seja, o aumento do plasma em relação aos demais componentes do sangue ${ }^{(15)}$.

O edema é um dos principais sinais do $\operatorname{VLE}^{(9-10,16)}$ e também considerado o mais sensível para tal inferência diagnóstica ${ }^{(11)}$. E, embora a anasarca tenha baixo percentual de manifestação, pode-se observar que tal característica ocorre mais em pacientes com VLE que em pacientes não portadores deste diagnóstico ${ }^{(16)}$, podendo ser considerada um sinal representativo do diagnóstico em questão.

Com relação às associações realizadas no presente estudo, é possível inferir que, quanto maior o tempo de internação na UTI, maior a suscetibilidade a eventos adversos, como o desenvolvimento do VLE, e maior a taxa de mortalidade ${ }^{(17)}$. $O$ revés também é válido: a ocorrência de eventos adversos aumenta o tempo de permanência da UTI, bem como a mortalidade, podendo conceber um ciclo ${ }^{(18)}$. Tanto a ventilação mecânica quanto a sedação têm impacto no tempo de internação na UTI(19-20). Porém, não há correlação entre nível de sedação e a permanência em ventilação mecânica ${ }^{(21)}$.

Assumindo que o balanço hídrico acumulado positivo é o mesmo que ingestão maior que eliminação, percebeu-se uma divergência de dados apresentados em literatura já existente. Enquanto o presente estudo identificou que o balanço hídrico acumulado positivo não tem associação significativa com o tempo de internação na UTI, a literatura preexistente traz significante associação entre a característica definidora "ingestão maior que a eliminação" e a variável "tempo de tratamento"(22).

A relação entre ventilação mecânica e sobrecarga hídrica justifica-se pela gravidade do quadro clínico apresentado pelo paciente, posto que pacientes com situação de saúde mais grave necessitam de ventilação mecânica e acabam também necessitando de maior infusão de fluidos, inclusive ressuscitação volêmica. Esta é comumente utilizada no primeiro dia de internação em UTIs. Além disso, quando a ressuscitação volêmica é efetuada com mais de $5 \mathrm{~L} /$ dia, está associada com aumento na mortalidade e aumento dos custos hospitalares, exceto quando se trata de pacientes não ventilados mecanicamente ${ }^{(23)}$.

O VLE pode resultar de múltiplos fatores, sendo importante uma avaliação clínica aprofundada que oriente o tratamento correto, que irá variar de paciente para paciente. Somente dados hemodinâmicos alterados, como por exemplo, o aumento da pressão venosa central, não basta para confirmar uma sobrecarga hídrica. É necessário analisar conjuntamente os sinais e sintomas apresentados, os dados hemodinâmicos alterados e até mesmo uso de biomarcadores ${ }^{(6)}$. Afinal, somente através de um diagnóstico acurado é possível planejar uma assistência de enfermagem resoluta ${ }^{(4)}$.

Para mais, sugere-se a realização de futuros estudos que supram as limitações de exclusividade de serviço e cenário de pesquisa e a impossibilidade de mensuração de alguns parâmetros hemodinâmicos (alteração na gravidade específica da urina, alteração da pressão arterial pulmonar, aumento da pressão venosa central e ganho de peso em curto período de tempo) que também podem evidenciar o VLE. 
A investigação trouxe novas contribuições acerca da inferência diagnóstica do VLE, para melhor direcionar o enfermeiro aos resultados e intervenções, bem como diminuir possíveis complicações, considerando a prevalência de $54,8 \%$ desse problema nos pacientes internados na UTI. Conclui-se que as características definidoras edema, hemoglobina e hematócrito diminuídos, anasarca e desequilíbrio eletrolítico, são indicadores clínicos que permitem a acurácia diagnóstica de VLE. Comprometimento neurológico, ventilação mecânica, sedação, e mais de quatro dias de internação foram evidenciados como principais fatores relacionados à causa desse diagnóstico em pacientes graves.

Os resultados também ampliam a possibilidade de desenvolvimento desse diagnóstico de enfermagem. Almeja-se encaminhar à apreciação da NANDA-I para a reformulação dos fatores relacionados e consolidação de características definidoras prevalentes.

\section{REFERÊNCIAS}

1. Kotz M, Frizon G, Silva OM da, Toniollo CL, Ascari RA. Tecnologias, humanização e o cuidado de Enfermagem na Unidade de Terapia Intensiva: uma revisão bibliográfica. Rev. Uningá Review. [Internet]. 2014 [acesso em 02 fev 2020]; 18(3). Disponível em: http://revista.uninga.br/index.php/uningareviews/ article/view/1512/1127.

2. Dutra HS, Jesus MCP de, Pinto LMC, Farah BF. Utilização do processo de enfermagem em unidade de terapia intensiva: revisão integrativa da literatura. HU Revista. [Internet]. 2016 [acesso em 02 fev 2020]; 42(4). Disponível em: http://ojs2.ufjf.emnuvens.com.br/hurevista/article/view/2413/901.

3. Silva RS da, Lima M de OM, Bandeira WC de O, Pereira AT, Sampaio AAC, Paixão GP do N. Prevalent nursing diagnosis in patients hospitalized in intensive care unit: an integrative review. Rev Enferm.

Contemp. [Internet]. 2016 [acesso em 02 fev 2020]; 5(2). Disponível em: http://doi.org/10.17267/23173378rec.v5i2.1023.

4. Herdman TH, Kamitsuru S. Diagnósticos de enfermagem da NANDA-I: definições e classificação 20182020. Porto Alegre: Artmed; 2018.

5. Ávila MON, Rocha PN, Zanetta DMT, Yu L, Burdmann E de A. Water balance, acute kidney injury and mortality of intensive care unit patients. J. Bras. Nefrol. [Internet]. 2014 [acesso em 02 fev 2020]; 36(3).

Disponível em: https://doi.org/10.5935/0101-2800.20140054.

6. Miller WL. Assessment and anagement of volume overload and congestion in chronic heart failure: can measuring blood volume provide new insights. Kidney Dis. [Internet]. 2016 [acesso em 02 fev 2020]; 2(4). Disponível em: https://doi.org/10.1159/000450526.

7. Albuquerque JM de, Silva RFA da, Souza RFF de. Epidemiological profile and monitoring after discharge of patients hospitalized at an Intensive Care Unit. Cogitare enferm. [Internet]. 2017 [acesso em 02 fev 2020]; 22(3). Disponível em: https://doi.org/10.5380/ce.v22i3.50609.

8. Zink E. Lesões Cranianas. In: Morton PG, Fontaine DK. Cuidados críticos de enfermagem: uma abordagem holística. Rio de Janeiro (RJ): Guanabara Koogan; 2007. p. 851-72.

9. Martins CP, Brandão MGSA, Freire MTJ, Marques KM de AP. Diagnósticos de enfermagem em unidade de terapia intensiva: uma revisão integrativa. Rev. Aten. Saúde [Internet]. 2018 [acesso em 02 fev 2020]; 16(57). Disponível em: https://doi.org/10.13037/ras.vol16n57.5124.

10. Boery RNS de O, Barros ALBL de, Lucena A de F. Características definidoras do Diagnóstico de enfermagem: volume de líquidos excessivo. Rev Gaúcha Enferm. [Internet]. 2005 [acesso em 02 fev 2020]; 26(3). Disponível em: https://seer.ufrgs.br/RevistaGauchadeEnfermagem/article/view/4563/2490.

11. Fernandes MI da CD, Bispo M de M, Leite EMD, Lopes MV de O, Silva VM da, Lira ALB de C. 
Diagnostic accuracy of the defining characteristics of the excessive fluid volume diagnosis in hemodialysis patients. Rev. Latino-Am. Enfermagem [Internet]. 2015 [acesso em 02 fev 2020]; 23(6). Disponível em: https://doi.org/10.1590/0104-1169.0380.2649.

12. Oliveira ACNP de, Cruz ICF da. Gerenciamento de enfermagem na prevenção da hipervolemia em pacientes de alta complexidade: uma revisão sistemática de literatura. J. Spec. Nurs. Care [Internet]. 2013 [acesso em 02 fev 2020]; 6(1). Disponível em: http://www.jsncare.uff.br/index.php/jsncare/article/ view/2468/582.

13. Fernandes MI da CD, Bispo M de M, Leite EMD, Lopes MV de O, Silva VM da, Lira ALB de C. Diagnostic accuracy of the defining characteristics of the excessive fluid volume diagnosis in hemodialysis patients. Rev. Latino-Am. Enfermagem [Internet]. 2015 [acesso em 02 fev 2020]; 23(6). Disponível em: https://doi.org/10.1590/0104-1169.0380.2649.

14. Martins QCS, Aliti GB, Linhares JC, Rabelo ER. Excess fluid volume: clinical validation in patients with decompensated heart failure. Rev. Latino-Am. Enfermagem [Internet]. 2011 [acesso em 02 fev 2020]; 19(3). Disponível em: https://doi.org/10.1590/S0104-11692011000300013.

15. Oriani G, Pavesi M, Oriani A, Bollina I. Acute normovolemic hemodilution. Transfus Apher Sci. [Internet]. 2011 [acesso em 02 fev 2020]; 45(3). Disponível em: https://doi.org/10.1016/j. transci.2011.10.006.

16. Guimarães HCQCP, Barros ALBL de, Gutierrez MGR de. Identificação das características definidoras do diagnóstico de enfermagem excesso de volume de líquidos. Rev.Latino-Am. Enfermagem [Internet]. 2000 [acesso em 02 fev 2020]; 8(2). Disponível em: https://doi.org/10.1590/S0104-11692000000200011.

17. Nogueira L de S, Sousa RMC de, Padilha KG, Koike KM. Clinical characteristics and severity of the patients admitted to the public and private ICUS. Texto Contexto - Enferm. [Internet]. 2012 [acesso em 02 fev 2020]; 21(1). Disponível em: https://doi.org/10.1590/S0104-07072012000100007.

18. Roque KE, Tonini T, Melo ECP. Adverse events in the intensive care unit: impact on mortality and length of stay in a prospective study. Cad. Saúde Pública. [Internet] 2016 [acesso em 02 fev 2020]; 32(10). Disponível em: https://doi.org/10.1590/0102-311X00081815.

19. Oliveira ABF de, Dias OM, Mello MM, Araújo S, Dragosavac D, Nucci A, et al. Factors associated with increased mortality and prolonged length of stay in an adult intensive care unit. Rev Bras Ter Intensiva [Internet]. 2010 [acesso em 02 fev 2020]; 22(3). Disponível em: https://doi.org/10.1590/S0103507X2010000300006.

20. Silva DC da, Barbosa TP, Bastos AS de, Beccaria LM. Association between intensities of pain and sedation in intensive care patients. Acta Paul Enferm. [Internet] 2017 [acesso em 02 fev 2020]; 30(3). Disponível em: https://doi.org/10.1590/1982-0194201700037.

21. Bragança HCE, Rezende AAB, Reis GR, Magagnin J da LS, Miranda EF de, Rodrigues ESR, et al. Correlation between levels of sedation and mechanical ventilation. Rev Amazônia Science Health [Internet]. 2014 [acesso em 02 fev 2020]; 2(2). Disponível em: http://www.ojs.unirg.edu.br/index.php/2/ article/view/633/249.

22. Fernandes MI da CD, Soares $C$ de $S$, Tinôco JD de S, Delgado MF, Paiva M das GMN de, Lopes MV de $O$, et al. Excess fluid volume: sociodemographic and clinical analysis in haemodialysis patients. Rev bras enferm [Internet]. 2017 [acesso em 02 fev 2020]; 70(1). Disponível em: https://doi.org/10.1590/00347167-2015-0138.

23. Marik PE, Linde-Zwirble WT, Bittner EA, Sahatjian J, Hansell D. Fluid administration in severe sepsis and septic shock, patterns and outcomes: an analysis of a large national database. Intensive Care Med. [Internet] 2017 [acesso em 02 fev 2020]; 43. Disponível em: http://doi.org/10.1007/s00134-016-4675-y. 


\section{COMO REFERENCIAR ESTE ARTIGO:}

Bittencourt CM, Busanello J, Harter J, Garcia RP. Incidência de volume de líquidos excessivo em pacientes adultos sob cuidados intensivos. Cogitare enferm. [Internet]. 2021 [acesso em "colocar data de acesso, dia, mês abreviado e ano"]; 26. Disponível em: http://dx.doi.org/10.5380/ce.v26i0.72689.

Recebido em: 05/04/2020

Aprovado em: 22/10/2020

Editora associada: Luciana Alcântara Nogueira

Autor Correspondente:

Caroline Monteiro Bittencourt

Universidade Federal do Pampa - Uruguaiana, RS, Brasil

E-mail: carolcaroline.mb@hotmail.com

Contribuição dos autores:

Contribuições substanciais para a concepção ou desenho do estudo; ou a aquisição, análise ou interpretação de dados do estudo - CMB, JB

Elaboração e revisão crítica do conteúdo intelectual do estudo - CMB

Aprovação da versão final do estudo a ser publicado - CMB, JB, JH, RPG

Responsável por todos os aspectos do estudo, assegurando as questões de precisão ou integridade de qualquer parte do estudo - CMB

Copyright $\odot 2021$ Este é um artigo em acesso aberto distribuído nos termos da Licença Creative Commons Atribuição, que permite o uso irrestrito, a distribuição e reprodução em qualquer meio desde que o artigo original seja devidamente citado. 